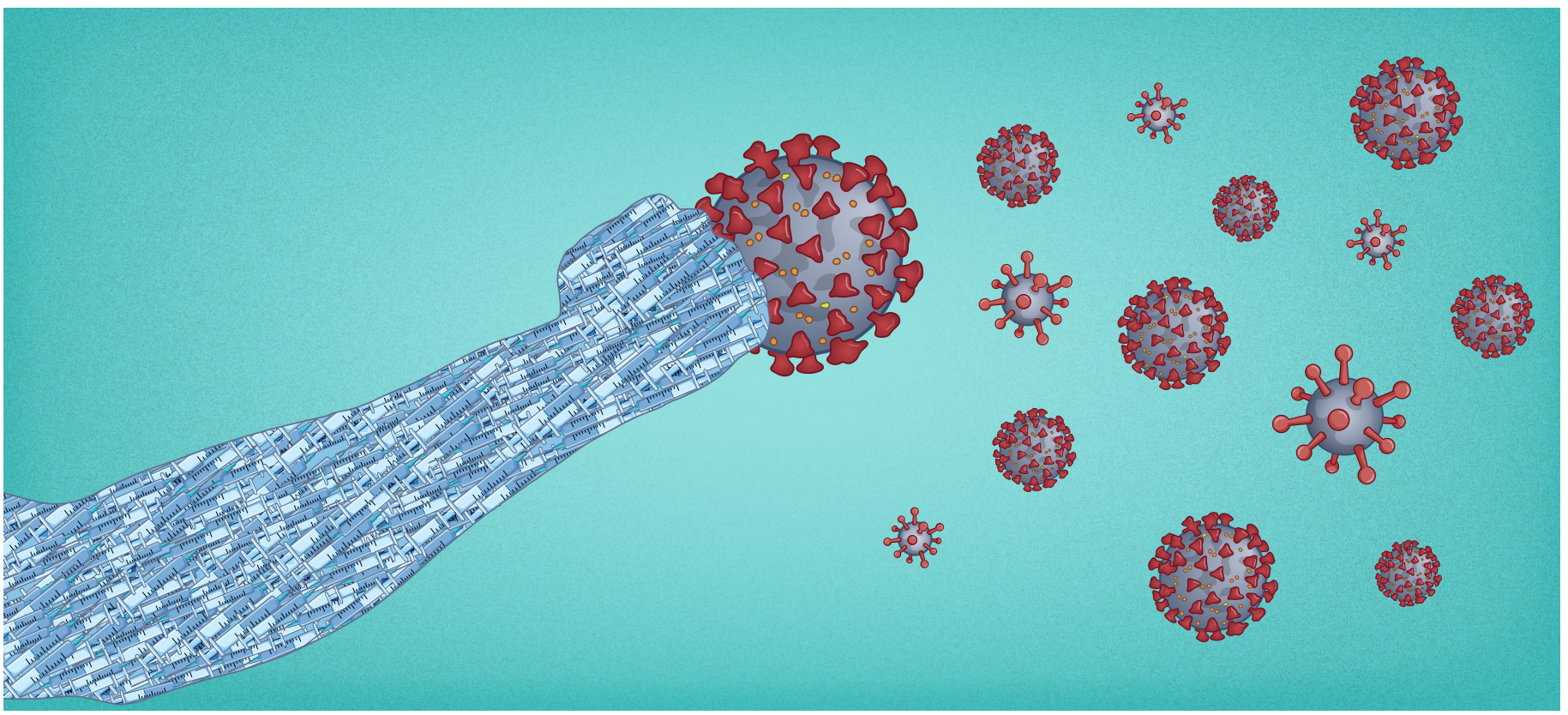

\title{
Preparing for the next plague
}

\author{
Whether pan-vaccines or antibodies, SARS-CoV-2 is adding impetus to the race for broad-spectrum \\ countermeasures against the world's next infectious scourges.
}

\section{Laura DeFrancesco}

\footnotetext{
n September, US president Joe Biden announced a plan to prepare for the next pandemic, with an initial outlay of $\$ 15$ billion and a total investment of $\$ 65.3$ billion over the next 10 years. The first goal is to "design, test, and approve a safe and effective vaccine against any pathogenic human virus within 100 days following the identification of an emergent viral pandemic." A series of steps are laid out to accomplish this, the first being to characterize a so-called prototype pathogen from each of 26 viral families known to infect humans, to help identify potential epitopes that could inform vaccine design.

Pandemic preparedness is hardly a new idea. The most recent of many such efforts was the Obama administration's pandemic playbook in 2016, following outbreaks of Ebola in West Africa and Zika in the Americas. In addition, the US National Institutes for Autoimmunity and Infectious Disease (NIAID) and the US Defense Advanced Research Projects Agency (DARPA) have had their sights on creating countermeasures that could be deployed quickly in the event of any outbreak. The speed with which the COVID-19 mRNA
}

vaccines were developed was not an accident, but can be traced back to work funded and directed by both agencies. Yet the success of the present vaccines was also somewhat serendipitous, as coronaviruses have a particularly easy target in their spike (S) protein, not to mention having been the subject of more than a decade of research after the SARS-CoV-1 outbreak in 2002 and Middle Eastern Respiratory Syndrome (MERS) in 2015. In the next pandemic, the world may not be so lucky.

So with a combination of optimism and fear-optimism that a fast response is possible and fear that the next pathogen might not succumb so easily-pandemic preparedness has taken on a renewed urgency, which hopefully won't dissipate once the current pandemic ends.

\section{Vaccines 2.0}

The teams working on mRNA vaccines for SARS-CoV-2 are already turning their attention to the next pandemic. They are trying to leverage the knowledge gained from the successes of first-generation SARS-CoV-2 vaccines for the next set of pandemic vaccines.
One advantage of the mRNA vaccine platform, according to Moderna's CSO Andrea Carfi, is that different mRNAs can be combined to target more than one pathogen or, in the case of SARS-CoV-2, more than one variant. Moderna has already announced that it has preclinical data on a combination flu and COVID-19 vaccine, which will be going into clinical trials next year, and it has plans for multivalent vaccines against SARS-CoV-2 variants beta and delta. There is no limit to the number of RNAs that can be combined, says Carfi. Moderna also has a cytomegalovirus (CMV) vaccine about to enter phase 3 trials that has six mRNAs, and has preclinical data on another comprising 10 mRNAs. Understanding how these mRNA cocktails give rise to an immune response is something the company is still working on. What its researchers can say is that they have tested multivalent vaccines encoding different proteins, and they can detect antibodies against the individual proteins. When it comes to timing, Carfi thinks they can go faster even than with their SARS-CoV-2 mRNA vaccine (which took 63 days from sequence selection to trial) 
by optimizing manufacturing and clinical readiness.

Given that the SARS-CoV-2 vaccine constituted the first experience of putting this type of vaccine in peoples arms on a global scale, safety had to be prioritized. But now, with hundreds of millions of people vaccinated, Carfi speculates that the US Food and Drug Administration (FDA) may have more confidence and familiarity with the platform, which could shave some time off the regulatory review process in future as well.

Ralph Baric's group at the University of North Carolina has been collaborating with the leading mRNA research groups from University of Pennsylvania, NIAID and Duke University. Their next-generation vaccine is a chimeric mRNA vaccine that builds on the modular nature of the coronavirus S protein. According to David Martinez, a postdoc in Baric's group since 2018, "The idea is to design a spike that instead of being monomorphic and eliciting immunity to one virus, you could increase the immunogenicity by having coverage for three viruses within one spike." This is possible because there are three sites that are the targets of protective antibodies-the $\mathrm{N}$-terminal domain, the receptor-binding domain (RBD) and the S2 domain. In a study published in Science, the Baric group report on a set of four different chimeric mRNA vaccines with different combinations of $\mathrm{N}$-terminal domain, RBD and S-protein mRNAs and show they can raise high levels of neutralizing antibodies against multiple sarbecoviruses (the subgenus of coronaviruses encompassing SARS-1 and SARS-2). A vaccine with only the SARS-CoV-2 S protein vaccine did not show the same breadth. One chimeric vaccine, however, raised antibodies against SARS-CoV, SARS-CoV-2, the SARS-CoV-2 beta variant and two bat coronaviruses (CoVRsShC014 and Cov1-WIV-1) that are thought to be poised for human emergence because they can replicate well in human primary airway cells. Although the level of antibodies was lower than with a monovalent vaccine, the breadth of coverage was greater.

Another way of going for breadth is to vaccinate with virus-like particles (VLP). According to Adam Simpson, CEO of Icosavax, a spinout from the University of Washington's Institute of Protein Design, "A VLP will inherently have a breadth of response that's different [from] a soluble protein whether it's made from a mRNA or not," However, not all viruses can be made into VLPs, and Icosavax's magic has been in using a computationally designed two-component system developed at the university, where any antigen can be displayed in an immunogenic array. The two components comprise proteins that are made separately and, when combined, self-assemble into a 128-subunit particle (VLP) with multifaceted icosahedral symmetry (imagine a soccer ball). The antigen (in the case of SARS-CoV-2, the $\mathrm{RBD}$ ) is linked to one of the proteins via a linker comprising 8,12 or 16 glycine and serine residues. "The reason it's so important," says Simpson, "is that it is a platform where we can put any antigen we want onto the VLP, and as components are proteins, they can be made by anyone with the technology for manufacturing proteins." In their case, the antigen is made in a mammalian cell line ( $\mathrm{CHO}$ cells) so that it's properly glycosylated, and the second component in Escherichia coli because it's inexpensive. "If you can make a protein, you can make our vaccine," Simpson says.

Although other mRNA and protein nanoparticle vaccines are multiplexing to get breadth of protection, Simpson thinks that won't be necessary with their VLPs. "If they look and smell like viruses, the body will react," he says. In 2020 preclinical work with a SARS-CoV-2 VLP displaying 60 RBDs, conducted at the University of Washington, Neil King's group found the VLPs not only produced tenfold higher titers than the $S$ protein (a version engineered to stabilize the protein in the form it has before fusing with a cell it infects, employed in COVID vaccines) but also targeted multiple epitopes, suggesting that it would be hard for the virus to mutate around the VLP vaccine, which protects against related strains not in the vaccine. With a $\$ 10$-million grant from the Gates Foundation and a partnership with Amgen, which is providing one of the protein components of the SARS-CoV-2 VLP, Icosavax has advanced VLP IVX-3441 into a phase $1 / 2$ clinical trial in Australia. A different VLP provided by University of Washington researchers is being tested in clinical trials by SK Bioscience.

Also at the University of Washington, David Veesler's group, which participated in the discovery work behind some anti-SARS-CoV-2 monoclonal antibodies being developed by Vir Biotechnology as well as Icosavax's VLP, continues to investigate how understanding of the basis of immunity to infectious agents can be used to guide vaccine design. In recently published work from his group, multivalent, mosaic VLPs and cocktails of different RBD VLPs raised neutralizing antibodies against a range of coronavirus variants and protected mice in challenge experiments with SARS-CoV-1, even when the combination did not include its RBD (Fig. 1). Veesler calls this an example of a second-generation

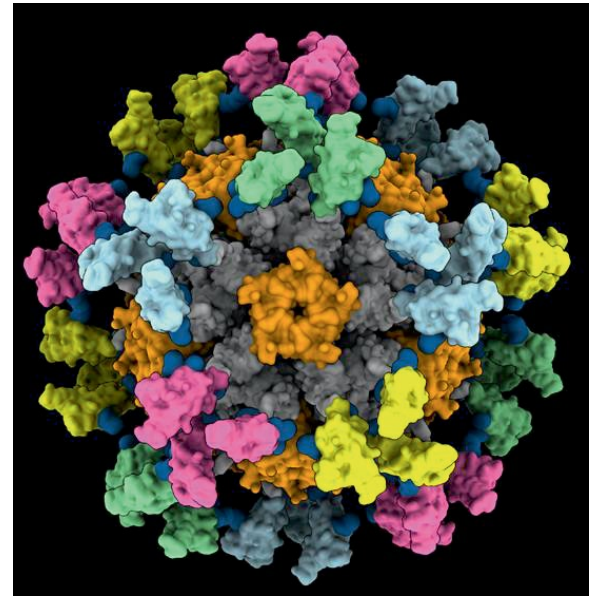

Fig. 1 | Structure of a multivalent SARS-CoV-2 spike receptor-binding domain nanoparticle vaccine. Modified with permission from Walls, A. C. et al. Cell 184, 5432-5477 (2021), Elsevier.

vaccine, vaccine 2.0 , which would be broadly neutralizing for multiple variants of SARS-CoV-2 and other sarbecoviruses. Vaccine 3.0 would be for beta-coronaviruses, which encompasses multiple lineages of which sarbecoviruses are only one. This is a long way off, however, due to the large diversity in the family, says Veesler.

"I'm careful not to use the term pan-sarbecovirus. There are reasons for that: there's a lot of diversity among known sarbecoviruses and we know that we might have only scratched the surface. There are so many that we have not yet discovered."

\section{Accelerated aspirations}

The Coalition for Epidemic Preparedness Innovations (CEPI) aims to accelerate the development of vaccines against emerging infectious diseases. Part of its rationale is to counter the boom-and-bust cycle of commercial development programs, which receive an influx of funding at the outset of an outbreak but historically run out of funding when the epidemic threat recedes. The CEPI was launched at Davos in 2017 after the Ebola epidemic in West Africa had killed $>11,000$ people; here, again, vaccine development came too late to save any lives (Fig. 2). Since its founding, the CEPI has supported a number of innovative vaccine programs, as well as some conventional ones.

In response to the current epidemic, the group has put $\$ 200$ million dollars on the table to start the race towards broadly protective vaccines both against SARS-CoV-2-this call ended in September, and applications are under review-and one for beta-coronaviruses (the family 


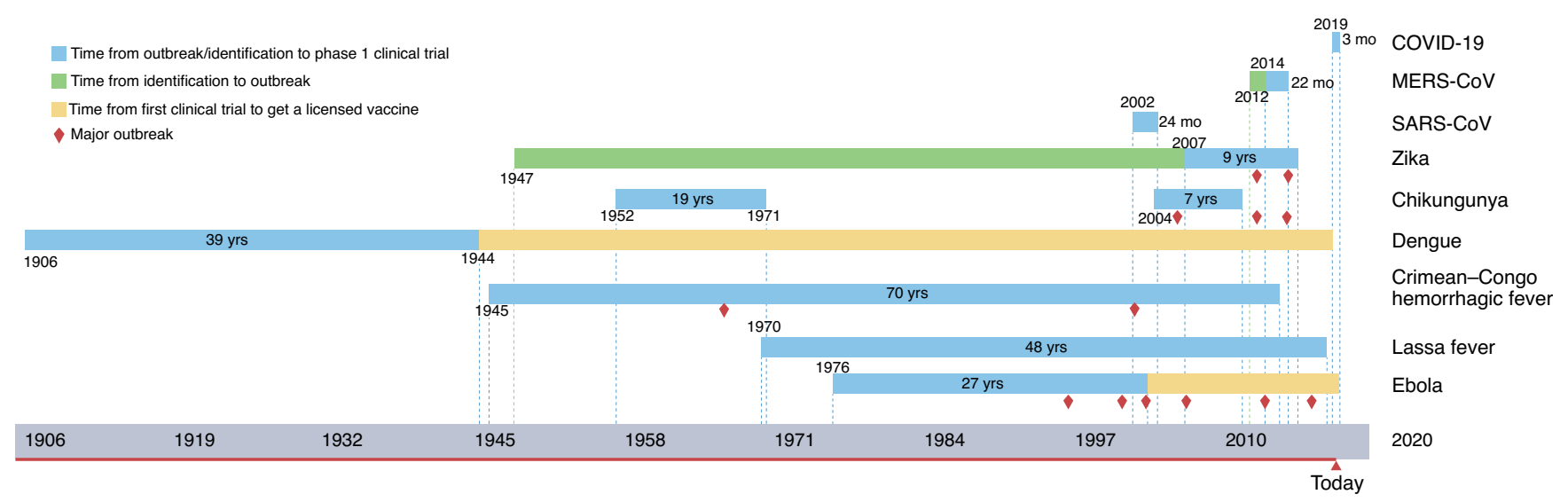

Fig. 2 | Emerging disease vaccine development timelines. Modified with permission from Kim, Y. C., Dema, B. \& Reyes-Sandoval, A. NPJ Vaccines 5, 34 (2020), Springer Nature.

that encompasses MERS as well as SARS viruses) more generally, which closed on 1 October 2021. “There's an awful lot of room for improvement, not just stability, which has been well covered in the media, but also the productivity, driving down the costs, improving the safety profile and potentially improving the longevity of the response," says Nick Jackson, the CEPI's head of programs and innovations.

In the United States, DARPA recognized that traditional vaccine development timelines prevent vaccines from being an effective countermeasure in a sudden outbreak. In 2012, it launched a five-year program, ADEPT-PROTECT (Autonomous Diagnostics to Enable Prevention and Therapeutics), to develop alternative technologies. Initially this set out to explore nucleic-acid-based therapies rather than the more traditional types based on inactivated viruses or recombinant protein subunits. In fact, the first clinical trial using a systemically administered mRNA-based therapeutic encoding a secreted protein, Moderna's monoclonal antibody (mAb) to chikungunya virus, was developed with DARPA backing (the company funded its phase 1 trial). The research behind that therapy came from another DARPA-supported group, Vanderbilt's Vaccine Center, which is directed by James Crowe. Crowe's group isolated potent neutralizing antibodies from a previously infected person, from which they determined the genetic sequence, which was used as the template for the mRNA-encoded antibody. Using the same approach, Crowe's group has produced other protective antibodies, among them a cocktail of antibodies that inactivates Ebola virus and an antibody against Zika virus. In September 2019, Moderna reported phase
1 clinical trial results of its chikungunya therapy. A few months later, they, along with the rest of the world, turned their attention to SARS-CoV-2.

In March 2020, a mere 63 days after the company received the sequence of SARS-CoV-2, a Moderna mRNA vaccine, mRNA 1273, developed in partnership with the Vaccine Research Center at NIAID, became the first COVID-19 vaccine to enter clinical trials. This effort came about because of support from DARPA-a $\$ 25$-million grant in 2003 to develop mRNA vaccines, followed by $\$ 56$ million in 2020 to support manufacturing of the SARS-CoV-2 mRNA vaccine. DNA vaccine company Inovia was the second company to trial a nucleic-acid-based COVID-19 vaccine, in April 2020, with backing from the Bill and Melinda Gates Foundation and DARPA. After some fits and starts (the FDA imposed a clinical hold due to problems with the device used to deliver the DNA), this vaccine (INO4800) received authorization to conduct phase 3 trials in Mexico and the United States.

\section{Antibodies as first responders} In 2017, as the ADEPT program was winding down, a follow-on program was spawned, the Pandemic Prevention Platform or P3. This program set up a challenge: to produce a protective antibody against a virus within 60 days of receiving a sample. According to P3's program manager, Amy Jenkins, "We envisioned that the likely approach would be a nucleic-acid-based antibody that would be similar to vaccines. It would deploy as an RNA-based antibody, which would turn the body into a bioreactor." Four teams took up the challenge: AbCellera Biologics, MedImmune/AstraZeneca, Duke University and Crowe's group at Vanderbilt University.
The groups practiced on various viruses, but then in 2019, halfway through the program, they were confronted with the real-life challenge of SARS-CoV-2. Crowe says that he and Jenkins contemplated whether they were ready to tackle this, especially as, early on, patient samples were unavailable. But as soon as a US patient was identified in January 2020), they decided to go for it. The first step-identifying an antibody-was achieved in roughly a week by AbCellera, followed by Vanderbilt. As AbCellera's CEO Carl Hansen described it, "We screened $\sim 6$ million B cells to identify $\sim 500$ unique antibodies that bound to the spike protein within a week."

As part of the $\mathrm{P} 3$ program, AbCellera had participated in a couple of capability demonstrations-pressure testing-that were critical leading up to the pandemic. "It allowed us build relationships and to figure out all the weak spots in handoffs and communication," says Hansen. Similarly, Vanderbilt's group had done a full Zika sprint in 78 days and was partway through sprints for an H3N2 and an H1N1 antibody when they pivoted to SARS-CoV-2. With lessons learned from these first two sprints, they went from receipt of convalescent patient blood sample in March 2020 (from individuals who had been infected in Wuhan, China, in December 2019) to the transfer of antibody sequences for validated potently neutralizing antibodies to AstraZeneca in 25 days.

At that point, Jenkins felt that technology for using nucleic acid vectors to deliver the mAbs was not far enough along in development to make an mRNA-encoded $\mathrm{mAb}$ at the scale required for an already out-of-control pandemic. "The more sure bet in the spring of 2020 was to take the antibodies into more traditional platforms. 
So that's what we did." AbCellera Biologics teamed up with Eli Lilly (which was not receiving P3 funding); and in May 2020, just 91 days after receiving the patient sample, they dosed their first patient. "They didn't hit 60 days, but we were only half way through the program," says Jenkins. Lilly received an Emergency Use Authorization (EUA) for bamlanivimab (a humanized IgG1 with modified Fc regions) for treating patients with mild to moderate COVID-19 in November 2020. "The encouraging thing was that they were quickly discovered, and among the first interventions along with remdesivir and some steroids-encouraging that we could deploy so rapidly, being honest, and surprised that protein-based manufacturing was quick," she says. Hansen gives credit to their partner, Lilly, for stepping up. "Lilly deserves tremendous credit for recognizing the opportunity. They haven't traditionally been in infectious disease and they wanted to be a positive force in responding to COVID," he says. Although the EUA for bamlanivimab as a monotherapy was revoked by the FDA in April 2021, it is still in use along with a second antibody, etesevimab, that Lilly licensed in from Shanghai Junshi Biosciences. Collectively these antibodies have been used to treat approximately 600,000 patients, according to Hansen. "The results have shown, if you look at the stats, [this] has probably saved tens of thousands of lives and tens of thousands of hospitalizations," he says.

From their COVID-19 sprints, Vanderbilt isolated hundreds of SARS-CoV-2 antibodies and licensed two to AstraZeneca, which engineered them to extend the half-life and to eliminate potentially harmful effector functions. AstraZeneca took AZD7442, a combination of two long-acting antibodies, tixagevimab and cilgavimab, that block the SARS-CoV-2 S protein from binding to its host receptor angiotensin-converting enzyme 2 (ACE2), into clinical trials for prevention of COVID19. Tixagevimab and cilgavimab are both human IgG1 mAbs engineered with five amino acid substitutions (at positions T240, M241, Y308, T310 and E312). In September, the company reported phase 3 clinical trial results on more than 3,000 at-risk (uninfected) people: the antibodies were $77 \%$ effective in preventing infection, making this the first $\mathrm{mAb}$ combination to demonstrate prevention of COVID-19-an important first in demonstrating a new way to enlist antibodies during a pandemic.

\section{Pain points}

The P3 groups collectively demonstrated that antibodies can be isolated and deployed quickly, not just for treatment but also for prevention, and that this can be done even from scratch in a few months, faster than for typical vaccines. And although the RNA vaccines were also put into arms in amazingly short order, a vaccine-induced immune response can take weeks to months to develop. Furthermore, RNA vaccines may not work for all viruses nor for all people, such as those who are immunocompromised (by some estimates, as many as 15 million in the US alone). For tamping down an emerging potential pandemic, antibodies potentially provide a better solution to contain an outbreak in its early days, while vaccine development and production proceed.

Historically, however, only a few anti-infective antibodies have ever been developed and approved for use. Two decades separated the approvals of the first two marketed anti-infective mAbs: palivizumab in 1999 and bezlotoxumab in 2017. The former is a humanized IgG1 $\kappa$ $\mathrm{mAb}$ targeting an epitope in the A antigenic site of the respiratory syncytial virus (RSV) F protein; the latter is a human IgG1 mAb that binds Clostridium difficile toxin $\mathrm{B}$

There are several reasons why such mAbs have not taken the world by storm. Much as for antibiotics, the commercial market for anti-infective antibodies has essentially failed. Most biotech companies working on $\mathrm{mAb}$ development have focused instead on more lucrative conditions in oncology, inflammatory disease or rare conditions. Any manufacturing base for an anti-infective $\mathrm{mAb}$ indicated for use in a pandemic must have the capacity to make antibodies for millions, or even billions, of people. Yet antibody manufacturing capacity, which typically uses mammalian (CHO) cells, is expensive and is finite worldwide. Even though the market is gargantuan, the high cost of goods for bulk manufacture of $\mathrm{CHO}$ cells means that anti-infective $\mathrm{mAbs}$ are not an attractive business proposition, as they have low product price points and low returns on investment. And the problems don't stop there: antibodies also pose problems for distribution in limited-resource public health settings because they must be administered by intravenous infusion, which requires hospitals or infusion centers and trained personnel, which very often are absent in resource-poor countries.

Several antibody engineering biotechs have been chipping away at these issues and have already started clinical testing (or will soon) of mAbs as prophylactics. Centivax, through a combination of dryand wet-lab techniques, has increased the mAb's half-life, broadened its delivery modes, increased its potency and removed potential unwanted effector functionsenabling, among other things, intramuscular (i.m.) injections of small amounts of their antibodies, which simplifies their administration and potentially drives down cost. Centivax founder Jacob Glanville applied his decades-long study of antibody structure-function relationships to understanding the properties of populations of complementarity-determining region (CDR) mutations that are found on naturally occurring antibodies after surviving selection. The Centivax team combined that bioinformatics information with phage display, where they can apply multifactor selection for features like high-affinity $\mathrm{mAb}$ binders and the lack of self-protein-protein interactions to avoid viscosity problems, among other properties. Centivax will be taking its first product, CENT-B9 for SARS-CoV-2, into clinical trials later this year, with funding from US Naval Medical Research Center, which has an interest in prophylactic antibodies to stop infections from spreading throughout a ship.

Adagio Therapeutics, which was spun out of the antibody engineering company Adimab in 2020 to fight the pandemic, has an engineered fully human $\mathrm{mAb}$ that reacts with all known SARS-CoV-2 variants now in phase $2 / 3$ trials for both disease treatment and prophylaxis. (Adagio is doing research and clinical development all on its own, having raised $>\$ 450$ million in venture capital and \$356 million in an initial public offering in 2021.) Tillman Gerngross, Adagio's founder and CEO, took a gamble that the pandemic was not going to go away quickly and embarked on a program of antibody design that would capture all the known and potentially unknown variants of SARS-CoV-2. "We knew we weren't going to win the race because it takes time to do all this engineering work. So from the beginning, we needed the best molecule with the expectation that it is going to be a longer haul and not just a pandemic that goes away, which is exactly how it played out," he says. Adagio's lead antibody, ADG20, was among a group isolated from a convalescent SARS-1 donor, and targets a conserved epitope on the S protein present on all SARS-1, SARS-2 and many potential emergent bat viruses. Using its antibody engineering platform, the company was able to retain breadth while improving binding affinity 500-fold and neutralizing capacity 70 -fold. ADG20 is the only small-molecule-like intramuscular injectable $\mathrm{mAb}$ that has this breadth, covering all SARS-CoV-2 variants, at such high affinity, according to Gerngross.

Crowe, although he directs Vanderbilt's vaccine center and is a believer in vaccines, thinks that i.m. delivery will be a game 
Table 1 | mAb therapies under development for prevention and/or prophylaxis

\begin{tabular}{|c|c|c|c|c|}
\hline Antibody (company) & Stage & Origin & Indication & Trial \\
\hline $\begin{array}{l}\text { Bamlanivimab + elesevimab } \\
\text { combination (Eli Lilly } \\
\text { AbCellera/Shanghai Junsai) }\end{array}$ & $\begin{array}{l}\text { EUA for post-exposure prophylaxis } \\
\text { September 2021; phase } 3 \text { (with NIAID) } \\
\text { for prevention in nursing home setting }\end{array}$ & $\begin{array}{l}\text { B cell from } \\
\text { convalescent } \\
\text { COVID-19 patients }\end{array}$ & Post-exposure prophylaxis & $\begin{array}{l}\text { BLAZE phase } 3 \text { (NIAID) } \\
80 \% \text { reduction in } \\
\text { nursing home setting } \\
\text { (NCT04497987) }\end{array}$ \\
\hline $\begin{array}{l}\text { Casirivimab and imdevimab } \\
\text { (Regeneron) }\end{array}$ & $\begin{array}{l}\text { Phase } 3 \text { for immunocompromised } \\
\text { patients; EUA for post-exposure } \\
\text { prophylaxis issued November } 2020\end{array}$ & $\begin{array}{l}\text { Humanized mice } \\
\text { and convalescent } \\
\text { plasma }\end{array}$ & $\begin{array}{l}\text { Pre-exposure prophylaxis in } \\
\text { at-risk individuals }\end{array}$ & NCT05074433 \\
\hline $\begin{array}{l}\text { AZD7442 (AZD8895 + } \\
\text { AZD1061 combination) } \\
\text { (AstraZeneca) }\end{array}$ & $\begin{array}{l}\text { Phase } 3 \text { completed/BLA for EUA for } \\
\text { pre-exposure prophylaxis (prevention) }\end{array}$ & $\begin{array}{l}\text { Convalescent } \\
\text { plasma from } \\
\text { COVID-19 patients, } \\
\text { engineered }\end{array}$ & $\begin{array}{l}\text { Pre- and post-exposure } \\
\text { prophylaxis }\end{array}$ & NCT04625725 \\
\hline ADG20 (Adagio) & Phase $2 / 3$ for prevention & $\begin{array}{l}\text { Engineered, } \\
\text { convalescent plasma } \\
\text { from COVID-19 } \\
\text { patients }\end{array}$ & Prevention in at-risk individuals & NCT04859517 \\
\hline Sotrovimab (Vir GSK) & $\begin{array}{l}\text { EUA for post-exposure prophylaxis; } \\
\text { approved and multiple EUAs for } \\
\text { treatment issued }\end{array}$ & $\begin{array}{l}\text { Convalescent } \\
\text { plasma from } \\
\text { COVID-19 patient }\end{array}$ & Prevention & \\
\hline CENT-B9 (Centivax) & $\begin{array}{l}\text { Phase } 1 \text { for safety, followed by phase } \\
2 / 3 \text { for prophylaxis and post-exposure } \\
\text { treatment }\end{array}$ & $\begin{array}{l}\text { Database of } \\
\text { natural antibodies, } \\
\text { engineered }\end{array}$ & $\begin{array}{l}\text { Pre- and post-exposure } \\
\text { prophylaxis and post-exposure } \\
\text { treatment }\end{array}$ & \\
\hline
\end{tabular}

EUA, Emergency Use Authorization; BLA, Biologics License Application. https://www.antibodysociety.org/covid-19-biologics-tracker/

changer for the use of mAbs in prophylaxis (Table 1). The question is how long they will protect against illness. The AstraZeneca trial went for three months and stopped when people started getting vaccinated. Crowe notes that he has been saying for years that for flu, an antibody-specifically one with a half-life extension to $\sim 90$ days, enabling protection potentially for up to a yearwould be a better alternative, as flu vaccines lose potency after a few months. "I get why people don't like saying this out loud. But this type of antibody could work better and longer," he says.

For pandemic preparedness, Herbert 'Skip' Virgin, executive vice president of research and CSO at the infectious disease company Vir Biotechnology, says: "What you want is an antibody that binds to as many of the viruses of a group as possible, that is insensitive to variation that's occurring in a pandemic or historically, and that is potent at a low dose as you can only manufacture so much, as you want to save as many lives as you can. These are the properties that are going to make the difference between a truly exceptional pandemic preparedness antibody and everything else. That's our philosophy, that's what we do and we think we have the antibodies that do this."

Vir's first commercial product, sotrovimab-a human IgG1к mAb engineered with an Fc domain of increased FcRn binding affinity that targets a conserved epitope on the SARS-CoV-2 S protein RBD-was granted full approval in Australia in August 2021, as well as EUAs in multiple countries. This $\mathrm{mAb}$, derived from $B$ cells of a patient who recovered from SARS-1 in 2003, targets an epitope on the S protein that is conserved in the sarbecovirus family. The antibody was engineered to extend its half-life, which, according to Virgin, providentially enhances distribution to the lung. Vir has a second $\mathrm{mAb}$ under development (VIR-7832) that in addition contains a mutation (with an Fc engineered to create M428L and N434S amino acid substitutions for increased human FcRn affinity) that has been shown in vitro to recruit effector functions. Virgin says that his team believes this offers the potential for the antibody to function as a T-cell vaccine, creating what they are calling dual-action antibodies. "This might not be the most popular way to word this-neutralization as a concept is easy to tell and easy to sell, but it's not accurate. There is no biological system in which neutralization is the only correlate of the efficacy of an antibody against viruses," he says. Sotrovimab is in trials now for i.m. administration, according to Bolyn Hubby, Vir's chief corporate affairs officer, which she says will be a "huge milestone in transitioning away from i.v."

More recently Vir researchers and collaborators have identified a number of antibodies with unique properties, among them broadly neutralizing activity targeting a cryptic epitope in the RBD, and a narrow escape profile (mutation scanning showed that only one substitution leads to escape).

\section{Down the road}

There are two approaches to pandemic preparedness: create platforms that can quickly be deployed; or have a stock of broadly effective products on the shelfantivirals, antibodies and vaccines. Several consortia are gearing up to do the latter.

Crowe's group at Vanderbilt came up with the idea they call 'Ahead 100', with the goal of making best-in-class antibodies for each of 100 targets from among the 25 families of viruses that are pathogenic to humans, to take them through a phase 1 safety trial with good manufacturing practice (GMP) material and stockpile 10,000 doses. He calculates that getting to that point will take $\$ 25$ million, so going beyond that for 100 antibodies is impractical for any single group. Instead, Crowe helped organize a consortium, the Global Pandemic Prevention and Biodefense Centerpotentially a $\$ 2.5$ billion project-which launched on 11 August 2021 to take this idea forward. The organization is housed in what is called the Connected DMV-Washington, DC, Maryland and Virginia-where there is a concentration of federal agencies $(\mathrm{NIH}$, FDA and Fort Detrick) and pharmaceutical company headquarters (AstraZeneca, GlaxoSmithkline and Emergent BioSolutions). Crowe raised $\$ 2.5$ million 
for the planning phase from the Gates Foundation, the CEPI, the US government and two commercial partners (Regeneron and Moderna). The consortium is looking for partners to put in \$25 million per target in the first project: Advanced Human Epidemic Antibody Defenses 100 (AHEAD 100), which will be led by Crowe.

The CEPI is thinking along the same lines. It is launching what Jackson described as "a world-class effort to rank all the families according to their threat level, in the most sophisticated way possible, by tapping into crowd-sourcing virologists, experts who understand zoonotic spillovers capable of using models and computational elements to derive a definitive list for what are the greatest threats across the families we know." Additionally, the coalition is working with a network of manufacturers to support the expansion of vaccine manufacturing capability around the world. "We want the regions to have equitable access and avoid the nationalisms and bilateral deals that have been problematic in this outbreak."

And the alphabet soup of US agencies are seemingly keeping their focus on the threat. DARPA has a new program called 'Nucleic Acids on Demand Worldwide' (NOW), which is looking to develop mobile manufacturing capacities that can be located where the need is greatest, and potentially shorten the time to distribute vaccines and therapies. In October, the NIAID announced a \$36-plus-million-dollar program to develop pan-coronavirus vaccines, with funding going to three academic programs, located at the University of Wisconsin, Madison, Brigham and Women's Hospital in Boston and Duke University in North Carolina. Recognizing the need for a comprehensive strategy, the funding is going to multidisciplinary groups with expertise in virology and immunology, immunogen design, and innovative vaccine and adjuvant platforms and technologies.

But preparing for the next coronavirus is not going to be enough. Nancy Sullivan, chief of the Biodefense Research Section at the NIAID, points out that even within a virus family, a single approach may not be a true prototype for the entire family. Her experience with filoviruses demonstrated this: "DNA vaccines protect against Marburg, but they don't protect against Ebola." What's needed, she points out, is "building a knowledge base, in a rationale way, and trying to incorporate some flexibility, not focusing on the hundreds of viruses that exist but instead focusing on the immunological principles that underpin protection and building that toolbox to cover those different immunological pathways." Something simple, such as the order in which you give prime and boost doses and the interval between them, can shape the immune response. "You can tune the immune response by adjusting one pair of vectors, which one you give first and the interval between the shots," she says.

Jeffry Ulmer, a retired vaccine expert with experience at Chiron, Merck, Novartis and GlaxoSmithKline, worries that the SARS-CoV-2 vaccine success may be the exception, rather than the rule, for future pandemics. "We saw a spectacular success with [mRNA vaccines for] COVID-19 but pretty much everything else that was tried also worked, including DNA. It ought to give us some pause that this particular antigen is relatively easy, and that the next application of the technology could face a much more difficult task."

\section{Laura DeFrancesco}

Senior Editor, Nature Biotechnology.

Published online: 19 November 2021 https://doi.org/10.1038/s41587-021-01140-X 